\title{
Probabilistic Multi-Factor Interaction Model for Evaluating Continuous Smooth Curves
}

\author{
Christos C. Chamis ${ }^{1}$ \\ NASA Glenn Research Center, Cleveland, OH 44135
}

\begin{abstract}
The Multi-Factor Interaction Model (MFIM) is used to evaluate the divot weight (foam weight ejected) from launch external tanks. The MFIM has sufficient degrees of freedom to evaluate a large number of factors that may contribute to the divot ejection. It also accommodates all interactions by its product form. Each factor has an exponent that satisfies only two points - the initial and final points. They are selected so that the MFIM will generate a common curve to fit the individual point data. The results show that the approach used generates the continuous curve.
\end{abstract}

\section{Introduction}

$\mathrm{T}$ HE simulation of complex material behavior resulting from the interaction of several factors (such as temperature, nonlinear material due to high stress, time dependence, fatigue, etc.) has been mainly performed by factor-specific representations. For example, entire text books are devoted to plasticity, creep, fatigue and high strain rate to mention only a few. Investigators have derived equations that describe material behavior for each factorspecific effect. Suppose we visualize that the material behavior is a continuum represented by some surface. Then, we can think of some representation which describes that surface which is inclusive of all participating factors that affect material behavior either singly or interactively in various combinations. To that end, research has been a continuing activity at NASA Glenn Research Center (GRC) for about thirty years. It started with a primitive form of the Multi-Factor Interaction Model (MFIM) representation for describing complex composite behavior in polymer matrix composites. ${ }^{1}$ It was extended to metal matrix composites ${ }^{2}$ and continued to be evolving during the National Aerospace Plane and the High Speed Research Programs. ${ }^{3}$ The result of all this research is the development of the MFIM to represent complex material point behavior by a single equation. ${ }^{4,5}$ The development of this equation starts with the premise that, if we are to quantify the range of factors affecting material point properties, we need a description of point behavior. ${ }^{6,7}$ In this context, it is reasonable to consider that behavior constitutes an $\mathrm{n}$ dimensional space (Point Behavior Space(PBS)) where each point on that surface represents a specific aspect of complex behavior. It is further reasonable to assume that PBS can be described by an assumed interpolation function. One convenient interpolation function is a polynomial of product form because mutual interactions among different factors can be represented by the overall product, and includes those cross products which are present in common algebraic polynomials.

\section{Approach}

Two samples are copied here, Figs. 1 and 2, from the previous paper ${ }^{8}$ to serve as an impetus to the present research. The results from using the MFIM are shown in Figs. 1 and 2. Note that the MFIM point-by-point simulation results match the point experimental data almost exactly. The reason for the research described in the present paper is to obtain a continuous curve (not linear) that will represent the data in the two figures. Least Squares generate a straight line and neural nets are not enough data to train the net. Therefore, an alternative is needed to generate a continuous curve which represents the data in Figs. 1 and 2. The present paper describes one approach on how to obtain continuous smooth curves by using the MFIM. The paper is in essence a continuation of last year's paper $^{8}$ where the approach was described on how to obtain point-by-point comparisons with the MFIM.

\footnotetext{
${ }^{1}$ Senior Aerospace Scientist, Research and Technology Directorate, 21000 Brookpark Road, Fellow.
} 


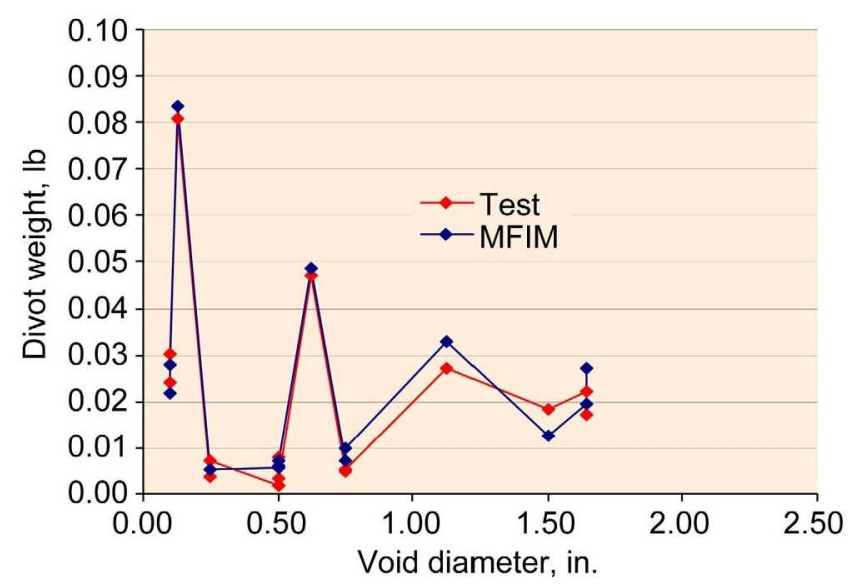

Figure 1. MFIM divot weight as a function of void diameter. (Cylindrical voids - cryo ingestion test).

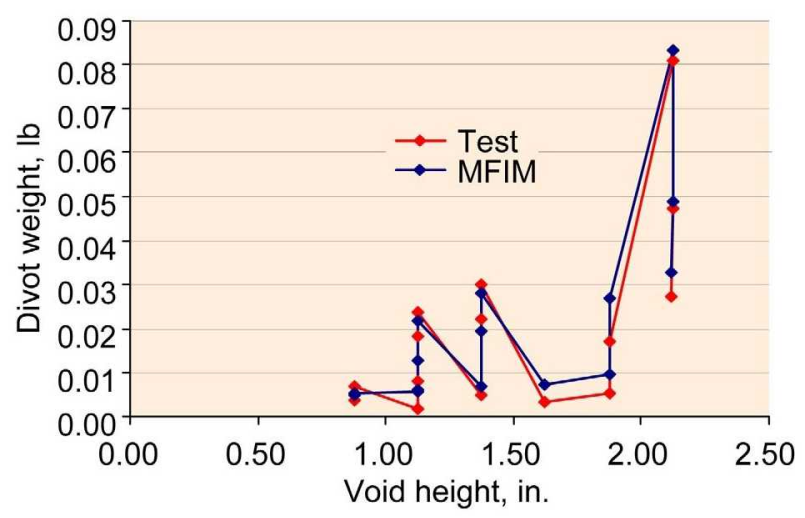

Figure 2. Probabilistic MFIM evaluation of divot weight. (Cylindrical voids—void ingestion test).

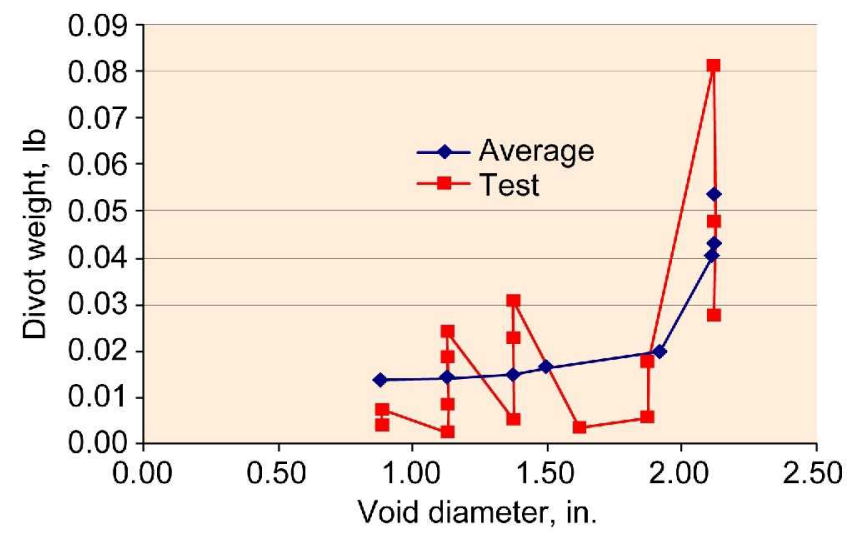

Figure 3. Continuous average curve for average probability versus void diameter.

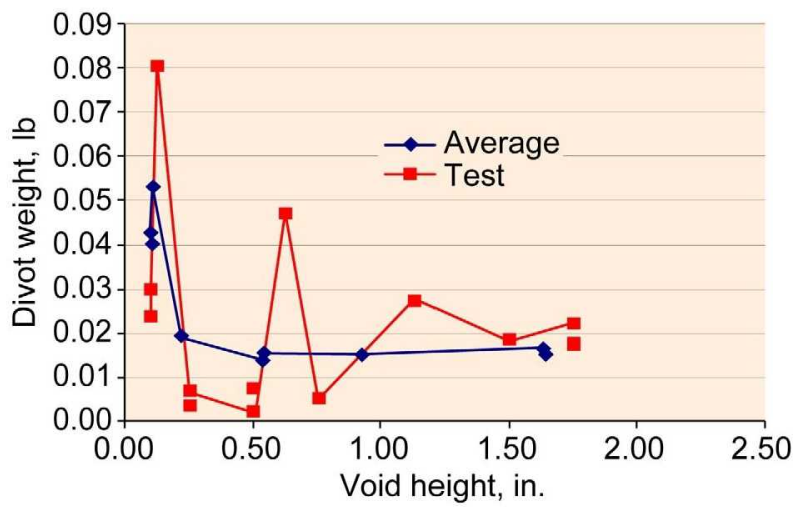

Figure 4. Continuous average curve for average probability versus void height.

The MFIM has the following form:

$$
\frac{W}{W_{o}}=\left[1-\left.\frac{x_{1}}{x_{1 f}}\right|^{e x 1}\left[1-\left.\frac{x_{2}}{x_{2 f}}\right|^{e x 2}\left[1-\left.\frac{x_{3}}{x_{3 f}}\right|^{e x 3} \cdots\left[1-\left.\frac{x_{n}}{x_{n f}}\right|^{e x n}\right.\right.\right.\right.
$$

Where $W$ is the predicted weight, $x_{1}, x_{2}, x_{3}$ are the variables, $x_{1 f}, x_{2 f}, x_{3 f}$ are their respective final values, and $e_{1}, e_{2}, e_{3}$ are the powers which are selected to satisfy the two conditions, the initial and final values. The exponent values are respectively, $-0.282,-0.131,-1.189$.

The approach is done in four specific steps. Step 1, the powers in the MFIM are evaluated. This will result in a point-by-point prediction, Figs. 1 and 2. Step 2, the highest points are evaluated providing a high continuous curve as are the lowest points providing a lower MFIM curve. Step 3, average points between the curves are selected and these average points are subsequently evaluated probabilistically, as shown by the continuous curves in Figs. 3 and 4. Step 4, the cumulative distribution function, Fig. 5, probability density function Fig. 6, and corresponding sensitivity factors, Fig. 7 are evaluated. 


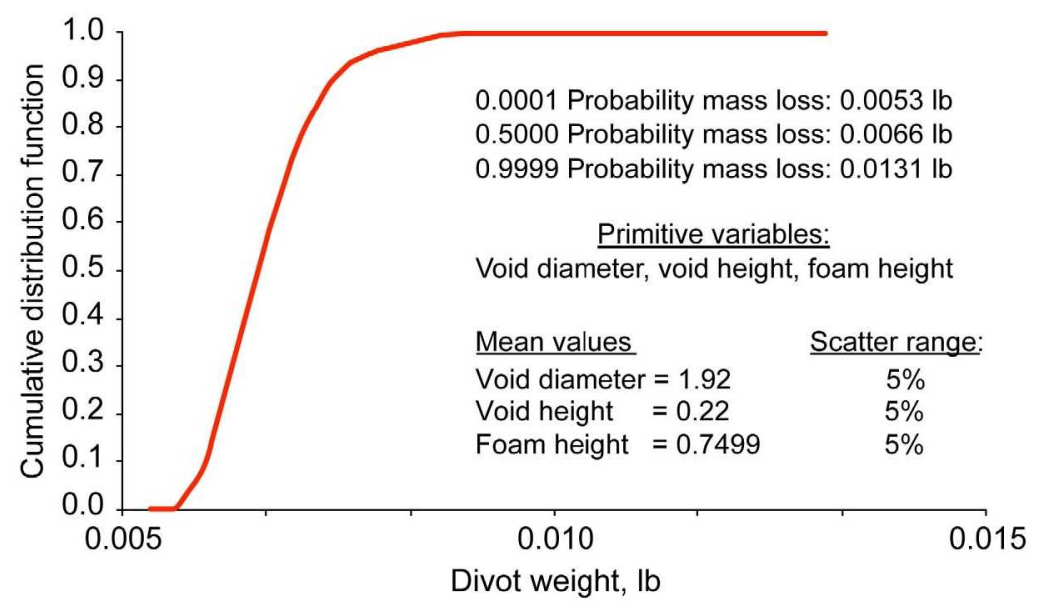

Figure 5. Probability cumulative distribution versus divot weight.

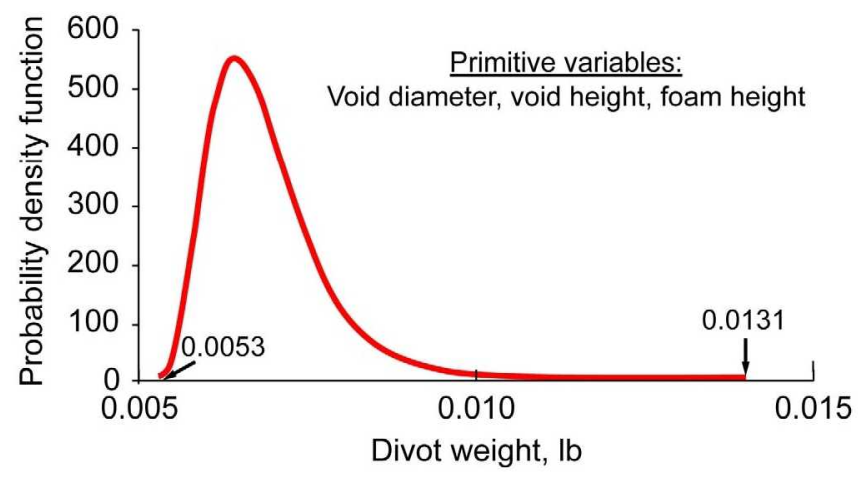

Figure 6. Probability density function versus divot weight.

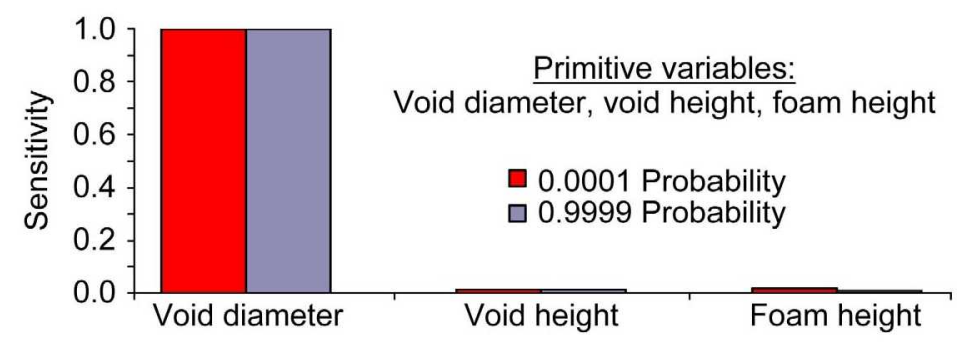

Figure 7. Sensitivity factors for low and high probability. 


\section{Results/Discussion}

The discussion is in two parts: Part 1, the MFIM fits and Part 2, the probabilistic simulation. Part I, the upper points maximum curve is fitted by MFIM as shown in Fig. 8. The curve was obtained by using only the upper points in that set. It is seen in Fig. 8 that only 3 points are used in the point-by-point fit by MFIM. The point-by-point fit obtained from the MFIM for the lowest set is shown in Fig. 9. It is seen in this figure that six points were fitted. Apparently there are more lower curve points than are for the upper curve. The minimum curve for the other set is shown in Fig. 10.

Note that only four points were used by the MFIM. A comparable result is shown in Fig. 11. Note that for this figure only five points were used for the MFIM fit. It is interesting to note how the average curves obtained by the MFIM compare with the average experimental data. This comparison is shown in Fig. 12 for the first set and in Fig. 13 for the second set. The fit is excellent as it should be because the average points were used in the fit.

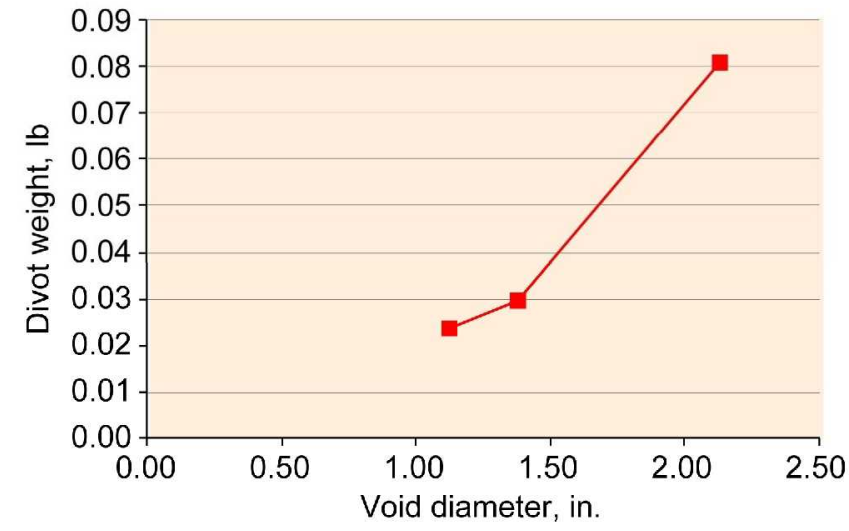

Figure 8. Continuous curve for maximum divot weight versus void diameter.

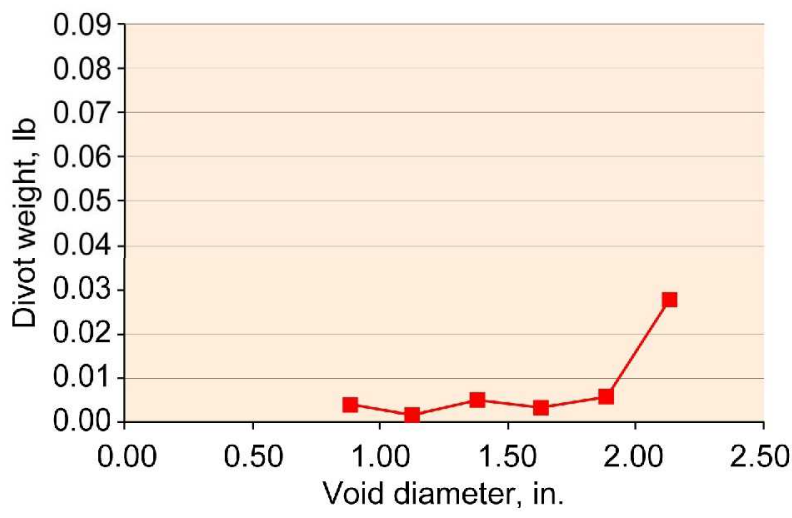

Figure 9. Continuous curve for minimum divot weight versus void diameter.

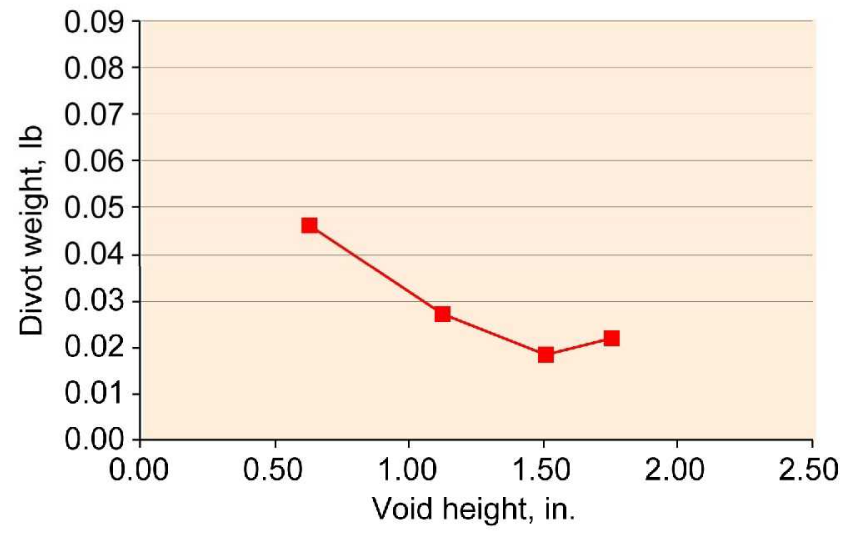

Figure 10. Continuous curve for maximum divot weight versus void height. 


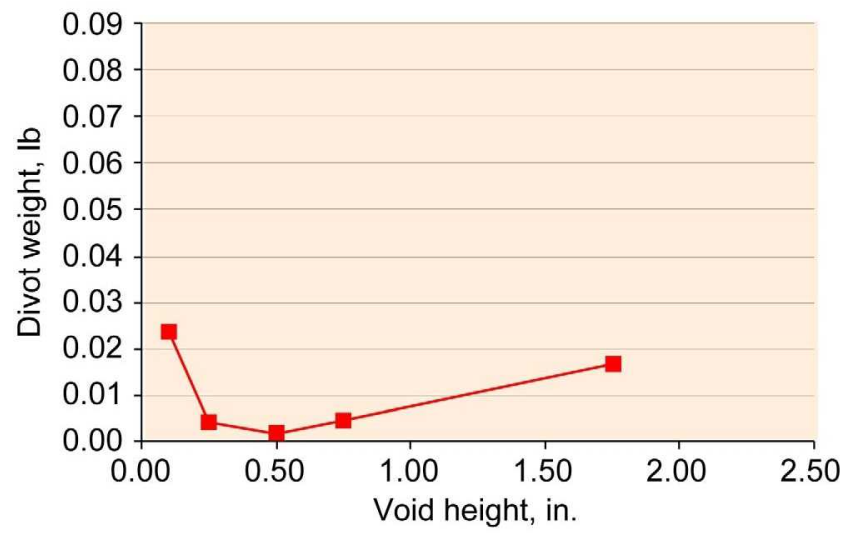

Figure 11. Continuous curve for minimum divot weight versus void height.

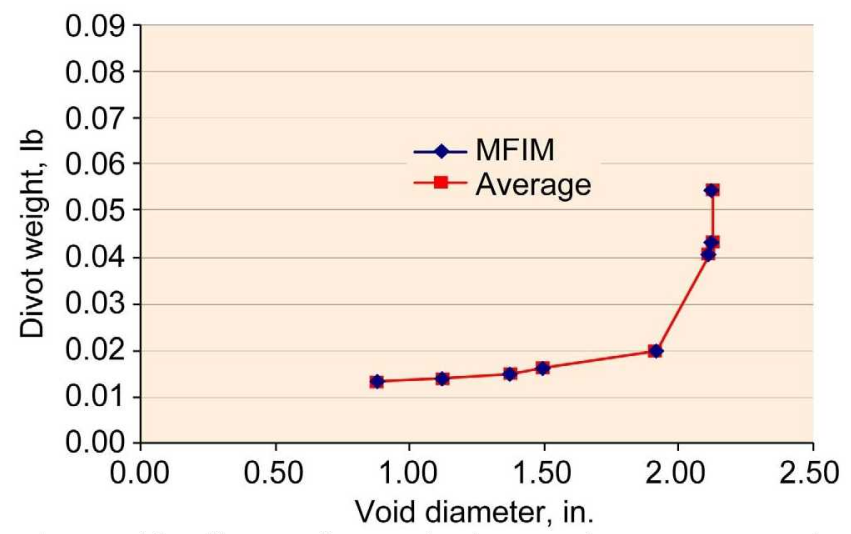

Figure 12. Comparison of divot weight versus void diameter obtained by MFIM and from the averages.

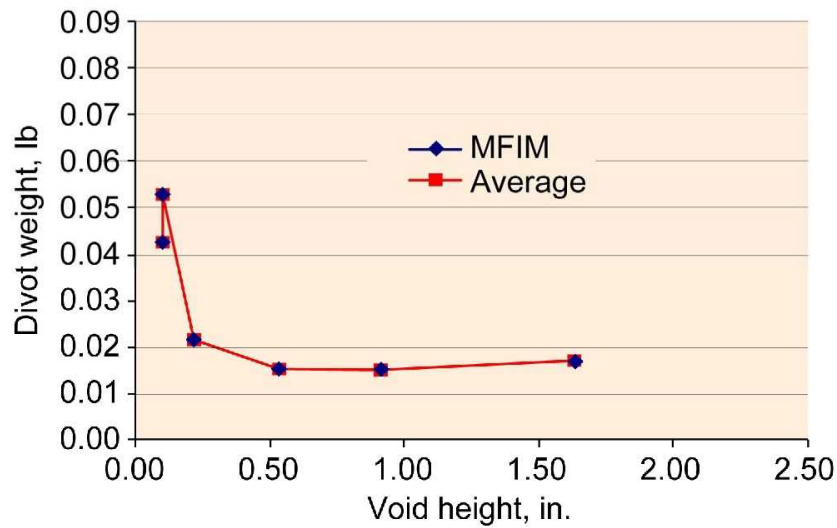

Figure 13. Comparison of divot weight versus void height obtained by MFIM and from the averages. 


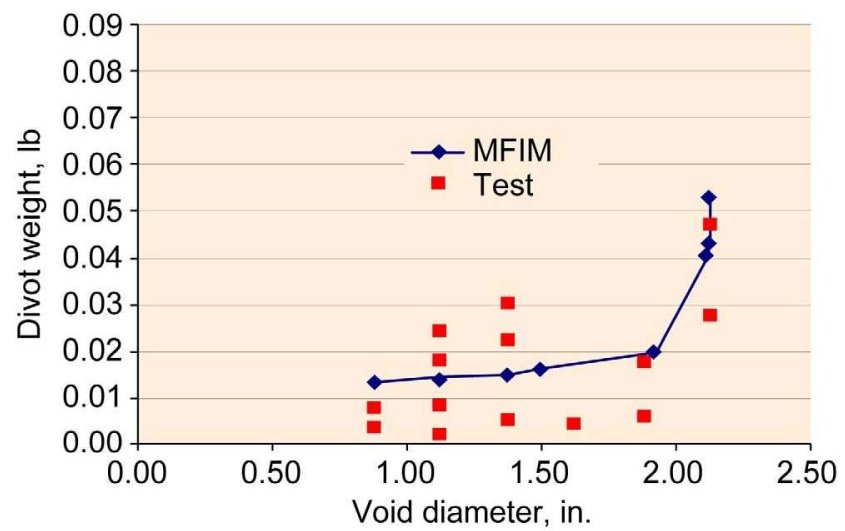

Figure 14. Combined curves predicted by MFIM versus void diameter superimposed on data.

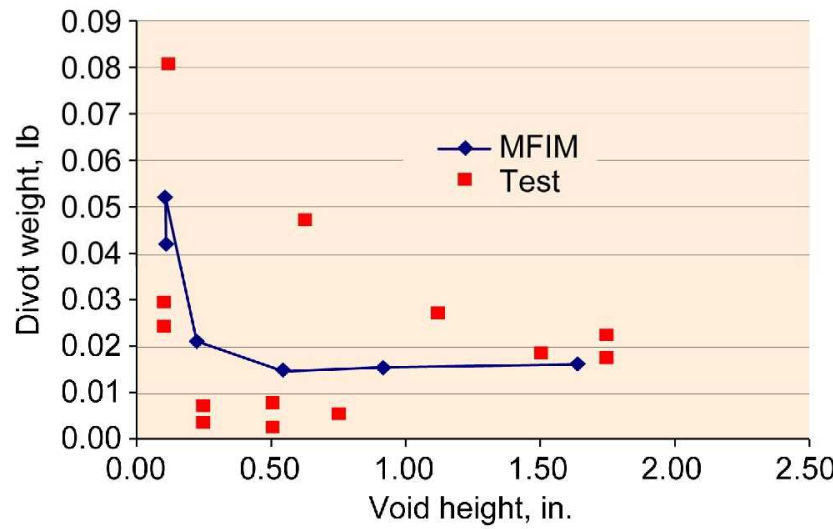

Figure 15. Combined curves predicted by MFIM versus void height superimposed on data.

Two more comparisons are instructive. The first one is shown in Fig. 14 for the comparison of the average curve predicted by the MFIM with all the experimental curve of the first set. A comparable comparison is shown in Fig. 15 for the experimental data of the second set. It is seen that in general no experimental data points are in the average curves. It is also interesting that the MFIM may be the only simulation that provides a reasonable averaged curve.

Part II. The probabilistic input information of numerical values to obtain the average curve is listed in Table 1. The numerical values for low probability of 1/10,000 and for high probability 9999/10,000 are listed in Table 2 .

Table 1. Input information for average curves

\begin{tabular}{|l|c|c|c|}
\hline \multicolumn{1}{|c|}{ Primitive variable } & Mean & $\begin{array}{c}\text { Coefficient of } \\
\text { variation, } \\
\text { percent }\end{array}$ & $\begin{array}{c}\text { Distribution } \\
\text { type }\end{array}$ \\
\hline Void diameter VD, (in.) & 1.9200 & 5 & Normal \\
\hline Void depth VH, (in.) & 0.2200 & 5 & Normal \\
\hline Foam height over void FH, (in.) & 0.7499 & 5 & Normal \\
\hline
\end{tabular}

Table 2. Numerical values for low and high probability

\begin{tabular}{|l|c|c|c|}
\hline \multicolumn{1}{|c|}{ Primitive variable } & $\begin{array}{c}\text { Starting } \\
\text { vector }\end{array}$ & $\begin{array}{c}0.0001 \\
\text { Probability }\end{array}$ & $\begin{array}{c}0.9999 \\
\text { Probability }\end{array}$ \\
\hline Void diameter VD, (in.) & 1.9200 & 0.736 & 1.070 \\
\hline Void depth VH, (in.) & 0.2200 & 0.120 & 0.1261 \\
\hline Foam over void FH, (in.) & 0.7499 & 0.280 & 0.300 \\
\hline
\end{tabular}


The probabilistic cumulative distribution function is shown in Fig. 5. The probability density function is shown graphically in Fig. 6. The respective sensitivity factors are shown graphically in Fig. 7. It can be seen in Fig. 5 that the curve rises rapidly and then levels off beyond the probability level of 0.99 . The probability density function shown in Fig. 6 has a very steep ascending part and equally a steep descending part to a level of about 0.0055 and then levels off approaching about to " $0.01+$ " at a value of 0.01 and continues at progressively lower values until 0.0131 . The sensitivity factors indicate that only the void diameter is the dominant variable in Fig. 7 . The probabilistic simulation demonstrates that the MFIM used in combination with a probabilistic simulation produces interesting and useful results. The cumulative distribution shows a probable scatter of mass loss of $0.0053 \mathrm{lb}$. at a probability level of 0.0001 or $1 / 10,000$ and about a loss of $0.131 \mathrm{lb}$. of a probability of $0.999 / 10,000$ which is equivalent to $0.060 \mathrm{lb}$. of scatter. The dimensions of the two dominant variables are listed in Table 2 for lowest and highest probability. Also shown in Tables 1 and 2 are values of the foam height over the void. This parameter has values at low and high probability. However, as the sensitivity factor indicates its influence was negligible as a result, no additional probabilistic information is available.

\section{Conclusion}

The salient results of using the multi-factor interaction model (MFIM) to obtain continuous curves when at few data points are available, as follows:

- The MFIM is a very efficient way to obtain average curves with limited data available (less than 10 data points).

- The MFIM in combination with probabilistic simulation provides additional information about the data, such as cumulative distribution and density functions and significant variables information.

- The combination also provides quantitative scatter as well as lowest and highest limits values.

- Probabilistic evaluation shows that the dominant variable influencing the weight is the void diameter.

- Probabilistic evaluation of the average MFIM shows that cumulative values and standard deviation are obtainable.

\section{References}

${ }^{1}$ Chamis, C. C., Lark, M. F., and Sinclair, J. H., "Integrated Theory for Predicting the Hygrothermo Mechanical Response of Advanced Composite Structural Components.” ASTM STP 658, 1978, pp. 160-192.

${ }^{2}$ Chamis, C. C. and Hopkins, D. A., "Thermoviscoplastic Nonlinear Constitutive Relationships for Structural Analysis of High Temperature Metal Matrix Composites." NASA TM-87291. November 1985.

${ }^{3}$ Chamis, C. C., Murthy, P. L. N. and Hopkins, D. A., "Computational Simulation of High Temperature Metal Matrix Composites Cyclic Behavior." ASTM, STP 1080, pp. 56-69.

${ }^{4}$ Tong, M. T., Singhal, S. N., Chamis, C. C. and Murthy, P. L. N., "Simulation of Fatigue Behavior of High Temperature Metal Matrix Composites." ASTM-Reprint from Standard Technical Publication 1253, 1996, pp. 540-551.

${ }^{5}$ Boyce, L. and Chamis, C. C., "Probabilistic Constitutive Relationships for Cyclic Material Strength Models." AIAA/ASME/ASCE/AHS $29^{\text {th }}$ Structures, Structural Dynamics and Materials Conference. Part 3, AIAA, 1988, pp. 1299-1306. 1996.

${ }^{6}$ Minnetyan, L., "Progressive Fracture Structural Analysis of National Wind Tunnel Structures." NASA CR-198485, May

${ }^{7}$ Chamis, C. C. and Minnetyan, L., "A Multi Factor Interaction Model for Damage Initiation and Progression." ASME/IMECE 2001/AD-25301, November 11, 2001.

${ }^{8}$ Chamis, C. C. and Abumeri, G. H., "Probabilistic Multi-Factor Interaction Model for Complex Material Behavior." AIAA/SDM Conference, Schaumberg, IL, April 7-10, 2008. 